Research Article

\title{
Mathematical Analysis of Nanostructured Surfaces: The Period-Scale Transform
}

\author{
Costas Poulios $(\mathbb{D})$ and Vassilios Constantoudis \\ Institute of Nanoscience and Nanotechnology, NCSR Demokritos, Nanometrisis P. C., Aghia Paraskevi 15310, Greece \\ Correspondence should be addressed to Vassilios Constantoudis; v.constantoudis@inn.demokritos.gr
}

Received 19 January 2021; Revised 6 May 2021; Accepted 12 June 2021; Published 22 June 2021

Academic Editor: Tianwei Zhang

Copyright (c) 2021 Costas Poulios and Vassilios Constantoudis. This is an open access article distributed under the Creative Commons Attribution License, which permits unrestricted use, distribution, and reproduction in any medium, provided the original work is properly cited.

\begin{abstract}
This work has been motivated by the urgent need for accurate and complete characterization of patterns consisting of almost periodic arrangements of specific features (trenches, bumps, holes, spikes, and so on) amply used in the industries of nanotechnology, microelectronics, and photonics. The quantitative characterization of such surface structures demands mathematical methods able to reveal both period- and feature-scale aspects. Given that the conventional approaches (Fourier or wavelet transform) are limited to either periodicity or feature-scale characterization, our work contributes with the proposal of a transformation which combines Fourier and wavelet merits to quantify simultaneously the period and feature scale of a periodic or almost periodic surface pattern. The output of our study has been (a) a detailed investigation of the mathematical properties of the proposed period-scale transform (PST) along with its relationship with other well-known transforms, (b) a presentation of some examples of PST of model 1D periodic surfaces to identify its benefits, and (c) first applications of PST in real profiles extracted from experimental polymer surfaces after plasma treatment.
\end{abstract}

\section{Introduction}

From the tiny scales of nanotechnology to the much larger feature sizes of geophysics and ocean studies, the morphology of surfaces plays an important role springing from its critical impact on surface properties and functionalities [1]. In order to understand and get control of these properties, it is necessary to find ways and tools to characterize surface morphology in a quantitative and concise way. A big concern in this endeavor is the presence of randomness and its co-existence with order on the arrangement of peaks and valleys on surfaces. Two extreme cases are the fully random surface where no point correlations exist and the fully periodic surface where a fixed surface unit is repeated endlessly along surface with a specific translation vector. More closely to the first case and usually met in nature is the scale-limited fractal (self-affine) surfaces where an anisotropic scale invariance in surface fluctuations prevails at small scales before being overwhelmed by uncorrelated randomness at larger scales. In technology, on the other side, and more specifically in nanotechnology where surface morphology can be controlled at nanometer scale, it is more common to fabricate surfaces either by top-down techniques or self-assembly processes with almost periodic structures consisting of similar humps or holes or trenches arranged in a periodic manner [2]. The same feature of (almost) periodicity is encountered in biological structures with an outstanding example-the surface morphology of moth eyes which has played a principal role in recent biomimetic technology [3]. Also, quite interestingly, recent studies have shown that periodic behaviour can be derived from stochastic mathematical models such as impulsive piecewise fractional functional differential equations or the stochastic CohenGrossberg neural networks with delays [4-6].

The most widely used method to detect and characterize periodicity of a surface is the Fourier transform (FT) and the diagram of the square of its amplitude versus frequency called power spectral density (PSD). Indeed, FT and mainly PSD are very sensitive to the presence of periodic repetition of a specific surface feature and display it with a well-defined peak at the frequency of repetition which is the inverse of period. Despite its wide and successful application to the 
detection and measurement of periodicity, PSD fails at the direct and straightforward extraction of the scale (width) of the repeated feature. This does not mean that PSD peak pattern is not sensitive to feature-scale modifications. These changes may affect the relevant PSD and therefore the number of well-defined peaks, but it is not always possible to get the real scales. For example, one can look at the $1 \mathrm{D}$ surfaces (Gaussian pulse trains) of Figures 1(a) and 1(b) both having the same period but repeated features with different scales. The PSDs shown in Figures 1(c) and 1(d) demonstrate the inability to map PSD peak changes to feature-scale values in a direct way despite the apparent effect of feature scale on the number of peaks.

FT provides a very accurate representation of the frequency content of a surface (or more generally of a signal). However, it does not have good localization properties. For instance, high jumps or holes in the surface cannot be read off easily from the Fourier transform. In many applications, this localization is desirable and can be first achieved by considering the windowed Fourier transform (WFT) (see [7] or [8]). WFT fixes a scale (the width of the window) and analyzes the surfaces from the point of view of this scale. The wavelet transform (WT) provides a similar description, but in contrast with WFT, the so-called "wavelets" do not have a fixed width but the width is adapted to the frequency. As a result, WT is a significant tool for analyzing short-lived phenomena such as singularities or steep and sudden scale changes. WT has found a plethora of profound applications including (but not limited to) geophysics (analysis of seismic data), engineering, and technology.

For instance, wavelet transform methods have been exploited in conditioning monitoring, that is, the process of monitoring some parameter or condition in machinery in order to detect any significant change that would be evidence for a developing default. This early detection and isolation of developing faults and the prediction of fault propagation allows for preventive maintenance and it can also serve as a countermeasure to the possibility of catastrophic incidence as a result of a failure. Furthermore, machinery and technological devices (for example wind turbines) are often subject to unexpected failures due not only to operational but also to environmental reasons. In such cases, prognosis techniques that can forecast the remaining life of machinery assure reliable production and lower maintenance costs. Several techniques have been developed in recent years towards this direction, which are based on WT, artificial intelligence, stochastic methods, and so on (see [9-12]). We also refer to the review [13] which investigates condition monitoring and fault diagnosis using various methods like wavelets and intelligent methods.

In summary, FT can be used to analyze the frequency content of a surface (or signal), but it has no localization properties and it cannot be used to detect the scale of the repeated feature of an almost periodic surface. On the other hand, the wavelet transform can capture the scale changes but cannot detect periodicity directly. Consequently, the need for a transform being capable of detecting and quantifying in a straightforward way in both aspects of periodicity (period and feature scale) is emerging. The aim of this paper is to propose and elaborate such a transform, which we name period-scale transform (PST). Its derivation is based on the periodization of a fundamental function satisfying some decay conditions. Periodization of functions has been studied extensively in analysis (see [14]), but, to the best of our knowledge, has not been used to define a transform.

This paper is organized as follows. In the next section, we define the transform rigorously, while in Section 3 we investigate its fundamental properties. In Section 4, we discuss its relevance to Poisson summation formula. The limit behaviour of the PST and the connection with other transforms (Fourier, wavelet, and Laplace) are the issues discussed in Sections 5 and 6, respectively. First examples of transform applications in selected model and real 1D surfaces are reported in Section 7 and the paper closes with summarizing the main conclusions in Section 8.

\section{Definition of the PS Transform}

We start with a non-zero function $g: \mathbb{R} \longrightarrow \mathbb{C}$ satisfying the decay condition

$$
|g(x)| \leq \frac{C}{(1+|x|)^{1+\varepsilon}},
$$

for any $x \in \mathbb{R}$, where $C, \varepsilon$ are positive constants. The function $g$ will be called the fundamental function. Given (scale) $s>0$ and (period) $\lambda>0$, we generate a periodic function $G(\cdot ; s, \lambda)$ as follows. Firstly, we change the scale of the function $g$, that is, we set $g_{s}(x)=g(x / s)$. Then, we apply the periodization operation to the function $g_{s}$ in order to obtain a periodic function with period $\lambda$. In other words, the function $G$ is defined as

$$
G(x ; s, \lambda)=\sum_{n=-\infty}^{\infty} g_{s}(x-n \lambda)=\sum_{n=-\infty}^{\infty} g\left(\frac{x-n \lambda}{s}\right) .
$$

The decay condition guarantees that the function $G(\cdot ; s, \lambda)$ is well defined. Furthermore, for any $x \in \mathbb{R}$, the following inequality holds:

$$
|G(x ; s, \lambda)| \leq 2 C+2 C\left(\frac{s}{\lambda}\right)^{1+\varepsilon} \sum_{n=1}^{\infty} \frac{1}{n^{1+\varepsilon}} .
$$

In some cases, the precise values of the constants are not of great importance. Consequently, instead of using (3), we often appeal to the following coarser inequality:

$$
|G(x ; s, \lambda)| \leq C+C\left(\frac{s}{\lambda}\right)^{1+\varepsilon},
$$

or even to the simple fact that, for $0<s \leq \lambda$, the (family of) function $(\mathrm{s}) G(\cdot ; s, \lambda)$ is bounded $|G(x ; s, \lambda)|<C$, where $C$ denotes some absolute constant (not always the same). Furthermore, we consider the mean value of the function $G$ in the interval $[0, \lambda]$ which will be denoted by $G$. That is,

$$
G=\frac{1}{\lambda} \int_{0}^{\lambda} G(x ; s, \lambda) \mathrm{d} x .
$$

An easy calculation shows that 


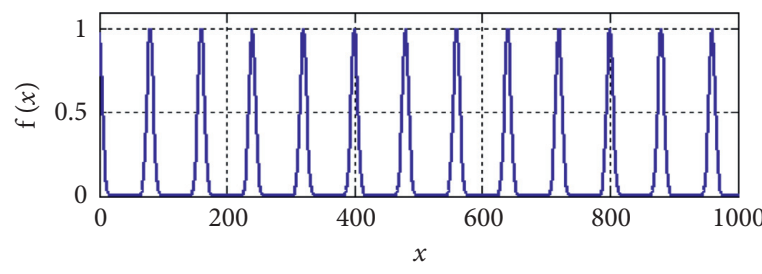

(a)

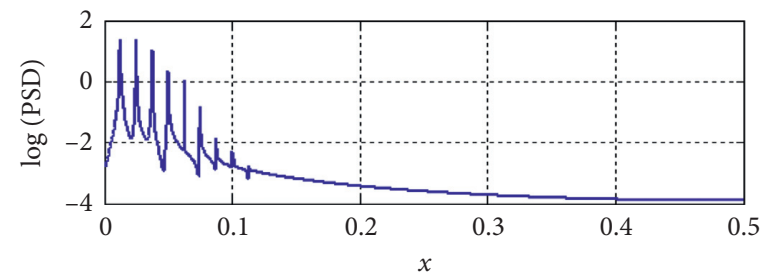

(c)

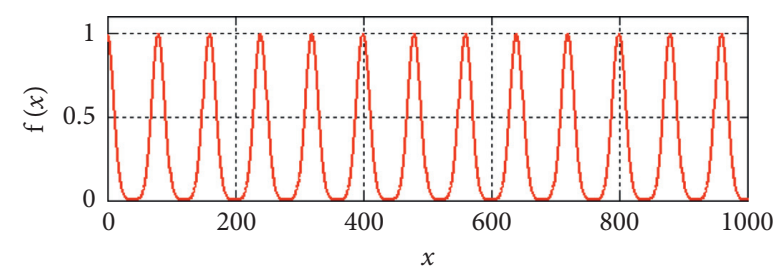

(b)

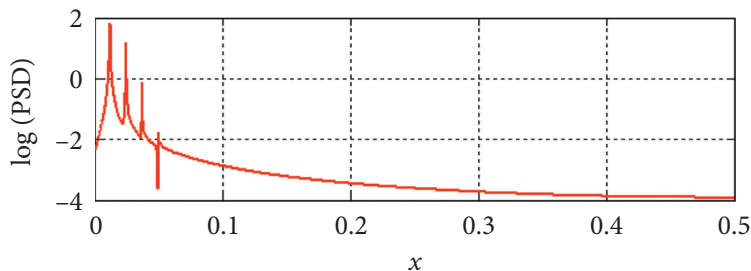

(d)

Figure 1: (a, b) Two profiles (Gaussian pulse trains) with identical periods (=80) but different peak widths (5 and 10, respectively). (c, d) The power spectra (PS) of the two profiles of (a) and (b) showing the difference in two cases: less peaks in profile of (b) with wider features. Despite the difference in the number of peaks in two power spectra, one cannot extract the real widths of profiles in a quantitative straightforward way.

$$
G=\frac{1}{\lambda} \int_{0}^{\lambda} \sum_{n \in \mathbb{Z}} g\left(\frac{x-n \lambda}{s}\right) \mathrm{d} x=\frac{1}{\lambda} \sum_{n \in \mathbb{Z}} \int_{0}^{\lambda} g\left(\frac{x-n \lambda}{s}\right) \mathrm{d} x=\frac{1}{\lambda} \sum_{n \in \mathbb{Z}} \int_{-n \lambda}^{-n \lambda+\lambda} g\left(\frac{x}{s}\right) \mathrm{d} x=\frac{1}{\lambda} \int_{-\infty}^{\infty} g\left(\frac{x}{s}\right) \mathrm{d} x=\frac{s}{\lambda} \int_{-\infty}^{\infty} g(x) \mathrm{d} x
$$

where interchanging the sum with the integral is justified by the dominated convergence theorem.

The function $G(x ; s, \lambda)-G$ is the kernel of the periodscale transform which is defined as follows.

Definition 1. Let $f: \mathbb{R} \longrightarrow \mathbb{R}$ be a function in $L^{1}(\mathbb{R})$. The period-scale transform (PS transform or PST in short) of $f$ with respect to the fundamental function $g$ is defined by

$$
\begin{aligned}
& \operatorname{PS}_{g} f:\{(s, \lambda) 0<s \leq \lambda\} \longrightarrow \mathbb{R} \\
& \operatorname{PS}_{g} f(s, \lambda)=\int_{-\infty}^{\infty} f(x)(G(x ; s, \lambda)-<G>) \mathrm{d} x .
\end{aligned}
$$

Since $G(\cdot ; s, \lambda)$ is bounded, for $f \in L^{1}(\mathbb{R})$, the function $f \cdot G$ also belongs to $L^{1}(\mathbb{R})$ and the above transform is well defined.

Additionally, using the dominated convergence theorem, we can analyze further the defining formula (7) of the PST and we obtain

$$
\begin{aligned}
\operatorname{PS}_{g} f(s, \lambda) & =\int_{-\infty}^{\infty} f(x)(G(x ; s, \lambda)-<G>) \mathrm{d} x=\int_{-\infty}^{\infty} f(x) \sum_{n=-\infty}^{\infty} g\left(\frac{x-n \lambda}{s}\right) \mathrm{d} x-<G>\int_{-\infty}^{\infty} f(x) \mathrm{d} x \\
& =\sum_{n=-\infty}^{\infty} \int_{-\infty}^{\infty} f(x) g\left(\frac{x-n \lambda}{s}\right) \mathrm{d} x-\frac{s}{\lambda} \int_{-\infty}^{\infty} g(x) \mathrm{d} x \int_{-\infty}^{\infty} f(x) \mathrm{d} x
\end{aligned}
$$

Remark 1. In principle, formula (7) (or, equivalently (8)) makes sense for any $s>0$ and $\lambda>0$, that is, the PST can be defined on the domain $(0, \infty) \times(0, \infty)$. However, given a function $f$, detecting and characterizing the periodicity and the scale feature of $f$ means that the scale $s$ is not bigger than the period $\lambda$. Hence, without losing important information for our purposes, the domain of $\mathrm{PS}_{g} f$ can be restricted to the set $\{(s, \lambda) 0<s \leq \lambda\}$.

\section{Properties of the PST}

3.1. Linearity and Boundedness. It is clear that the PST is linear. That is, for any $f, h \in L^{1}(\mathbb{R})$ and any scalar $\alpha$, we have

$$
\operatorname{PS}_{g}(f+h)=\operatorname{PS}_{g} f+\operatorname{PS}_{g} h \text { and } \operatorname{PS}_{g}(\alpha f)=\operatorname{PS}_{g} f \text {. }
$$

Furthermore, the PST defines a bounded linear operator 


$$
\mathrm{PS}_{g}: L^{1}(\mathbb{R}) \longrightarrow L^{\infty}(\{(s, \lambda): 0<s \leq \lambda\}) .
$$

Indeed, since $G$ is bounded, formula (7) gives that

$$
\begin{aligned}
\left|\mathrm{PS}_{g} f(s, \lambda)\right| & =\left|\int_{-\infty}^{\infty} f(x) \cdot G(x ; s, \lambda) \mathrm{d} x \mathrm{~d} x-\frac{s}{\lambda} \int_{-\infty}^{\infty} g(x) \mathrm{d} x \int_{-\infty}^{\infty} f(x) \mathrm{d} x\right| \\
& \leq \int_{-\infty}^{\infty}|f(x)| \cdot|G(x ; s, \lambda)| \mathrm{d} x+\frac{s}{\lambda} \int_{-\infty}^{\infty}|g(x)| \mathrm{d} x \int_{-\infty}^{\infty}|f(x)| \mathrm{d} x \\
& \leq C \int_{-\infty}^{\infty}|f(x)| \mathrm{d} x+\|g\|_{L^{1}}\|f\|_{L^{1}} \leq\left(C+\|g\|_{L^{1}}\right)\|f\|_{L^{1}} .
\end{aligned}
$$

3.2. Scaling Properties. Suppose that $f$ is a periodic function, for instance $f(x)=\sin (2 \pi x)$. Then, $f(2 x)=\sin (4 \pi x)$ is also a periodic function; however, both the period and the scale have been multiplied with the factor $1 / 2$. More generally, it is reasonable to expect that the PST of the function $f(t x), t>0$, is connected with the PST of the original function $f$ evaluated at the point $(t s, t \lambda)$. This is true and is given in the next theorem.

Theorem 1. Let $f \in L^{1}(\mathbb{R})$ and $t>0$.

(i) If $h(x)=f(t x)$, then the following holds:

$$
\mathrm{PS}_{g} h(s, \lambda)=\frac{1}{t} \mathrm{PS}_{g} f(t s, t \lambda) .
$$

(ii) If we set $\ell_{t}(f)(x)=1 / t f(x / t)$, then

$$
\mathrm{PS}_{g}\left(\ell_{t}(f)\right)(t s, t \lambda)=\mathrm{PS}_{g} f(s, \lambda) .
$$

Proof. By the definition of the PST, it follows that

$$
\begin{aligned}
\operatorname{PS}_{g} h(s, \lambda) & =\int_{-\infty}^{\infty} h(x) \sum_{n=-\infty}^{\infty} g\left(\frac{x-n \lambda}{s}\right) \mathrm{d} x-\frac{s}{\lambda} \int_{-\infty}^{\infty} g(x) \mathrm{d} x \int_{-\infty}^{\infty} h(x) \mathrm{d} x \\
& =\int_{-\infty}^{\infty} f(t x) \sum_{n=-\infty}^{\infty} g\left(\frac{x-n \lambda}{s}\right) \mathrm{d} x-\frac{s}{\lambda} \int_{-\infty}^{\infty} g(x) \mathrm{d} x \int_{-\infty}^{\infty} f(t x) \mathrm{d} x .
\end{aligned}
$$

Setting $t x=x^{\prime}$ implies that

$$
\operatorname{PS}_{g} h(s, \lambda)=\frac{1}{t} \int_{-\infty}^{\infty} f(x) \sum_{n=-\infty}^{\infty} g\left(\frac{x-n \lambda t}{t s}\right) \mathrm{d} x-\frac{s}{t \lambda} \int_{-\infty}^{\infty} g(x) \mathrm{d} x \int_{-\infty}^{\infty} f(x) \mathrm{d} x=\frac{1}{t} \mathrm{PS}_{g} f(t s, t \lambda)
$$

The second assertion follows immediately by the first one and the linearity of the PST.

Having dealt with dilation, we now turn our attention to translation. That is, given a function $f \in L^{1}(\mathbb{R})$ and any $y \in \mathbb{R}$, we are interested in finding the PST of the function $\tau_{y} f(x)=f(x-y)$.

Theorem 2. Let $f \in L^{1}(\mathbb{R})$ and $y \in \mathbb{R}$. The PST of the translation $\tau_{y} f$ of $f$ is given by

$$
\operatorname{PS}_{g}\left(\tau_{y} f\right)(s, \lambda)=\operatorname{PS}_{h} f(s, \lambda)
$$

where $h=\tau_{-y / s}(g)$.

Observe that the fundamental function $h$ in the righthand side of the above equation depends on $s$. In particular, this implies that the PST is not phase invariant.

Proof. It is not hard to see that the function $\tau_{-y / s}(g)$ satisfies a decay condition similar to (1), where the constant $C$ depends on $y / s$. Hence, it can be used as a fundamental function for the PST. Now, for fixed $s>0$ and $\lambda>0$, we observe that

$$
\begin{aligned}
\int_{-\infty}^{\infty} \tau_{y}(f)(x) G(x ; s, \lambda) \mathrm{d} x & =\sum_{n=-\infty}^{\infty} \int_{-\infty}^{\infty} f(x-y) g\left(\frac{x-n \lambda}{s}\right) \mathrm{d} x \\
& =\sum_{n=-\infty}^{\infty} \int_{-\infty}^{\infty} f(x) g\left(\frac{x+y-n \lambda}{s}\right) \mathrm{d} x \\
& =\sum_{n=-\infty}^{\infty} \int_{-\infty}^{\infty} f(x) \tau_{-y / s}(g)\left(\frac{x-n \lambda}{s}\right) \mathrm{d} x=\sum_{n=-\infty}^{\infty} \int_{-\infty}^{\infty} f(x) h\left(\frac{x-n \lambda}{s}\right) \mathrm{d} x
\end{aligned}
$$

Clearly, we have 


$$
\int_{-\infty}^{\infty} \tau_{y}(f)(x) \mathrm{d} x=\int_{-\infty}^{\infty} f(x) \mathrm{d} x, \int_{-\infty}^{\infty} h(x) \mathrm{d} x=\int_{-\infty}^{\infty} g(x) \mathrm{d} x,
$$

and the desired result follows immediately from equation (8).

In the special case $\lambda=y$, since $G(\cdot, s, \lambda)$ has period $\lambda$, it follows that translating $f$ by $\lambda$ brings no change to the PST. That is, we have the next result whose proof is straightforward.

Proposition 1. For any $f \in L^{1}(\mathbb{R})$ and $s>0, \lambda>0$, we have

$$
\mathrm{PS}_{g}\left(\tau_{\lambda} f\right)(s, \lambda)=\operatorname{PS}_{g} f(s, \lambda) \text {. }
$$

3.3. Continuity. The purpose of the present section is to show that the PST of any $f \in L^{1}(\mathbb{R})$ is a continuous function provided that $g$ is continuous. Firstly, we need the following lemma whose proof depends on elementary calculus arguments and it is omitted.
Lemma 1. Assume that the fundamental function $g$ is continuous. Then, the periodization $G$ of $g$ is continuous with respect to $(s, \lambda)$. That is, for any (fixed) $x \in \mathbb{R}$ and any $s_{0}>0$, $\lambda_{0}>0$, we have

$$
\lim _{(s, \lambda) \longrightarrow\left(s_{0}, \lambda_{0}\right)} G(x ; s, \lambda)=G\left(x ; s_{0}, \lambda_{0}\right) .
$$

Using the above lemma, we can prove the main result of this section.

Theorem 3. Assume that the fundamental function $g$ is continuous. Then, for any $f \in L^{1}(\mathbb{R})$, the PST of $f$ is continuous in $\{(s, \lambda) \mid 0<s \leq \lambda\}$.

Proof. Let $0<s_{0} \leq \lambda_{0}$ be fixed. Then, we have

$$
\left|\int_{-\infty}^{\infty} f(x) G(x ; s, \lambda) \mathrm{d} x-\int_{-\infty}^{\infty} f(x) G\left(x ; s_{0}, \lambda_{0}\right) \mathrm{d} x\right| \leq \int_{-\infty}^{\infty}|f(x)| \cdot\left|G(x ; s, \lambda)-G\left(x ; s_{0}, \lambda_{0}\right)\right| \mathrm{d} x .
$$

For $0<s \leq \lambda$, the family of functions $G(\cdot ; s, \lambda)$ is bounded by some absolute constant $C$ independent of $s$ and $\lambda$. Therefore, the dominated convergence theorem and the previous lemma imply that

$$
\lim _{(s, \lambda) \longrightarrow\left(s_{0}, \lambda_{0}\right)} \int_{-\infty}^{\infty}|f(x)| \cdot\left|G(x ; s, \lambda)-G\left(x ; s_{0}, \lambda_{0}\right)\right| \mathrm{d} x=0,
$$

and consequently,

$$
\lim _{(s, \lambda) \longrightarrow\left(s_{0}, \lambda_{0}\right)} \int_{-\infty}^{\infty} f(x) G(x ; s, \lambda) \mathrm{d} x=\int_{-\infty}^{\infty} f(x) G\left(x ; s_{0}, \lambda_{0}\right) \mathrm{d} x .
$$

By equation (8), it follows now that the PST of $f$ is a continuous function.

3.4. Other Forms of the PST. The aim of this section is to present some other forms of the PST which will be useful in the sequel. We start with the following proposition whose proof is straightforward.

Proposition 2. Assume that the function $f$ satisfies a decay condition similar to (1). Then, the PST of $f$ is given by

$$
\mathrm{PS}_{g} f(s, \lambda)=\int_{-\infty}^{\infty}\left(F_{\lambda}(x)-F_{\lambda}\right) g_{s}(x) \mathrm{d} x,
$$

where $F_{\lambda}(x)=\sum_{n \in \mathbb{Z}} f(x+n \lambda)$ is the periodization of $f$ with period $\lambda$ and $F_{\lambda}=1 / \lambda \int_{0}^{\lambda} F_{\lambda}(x) d x=1 / \lambda \int_{-\infty}^{\infty} f(x) d x$ is the mean value of $F_{\lambda}$ in the interval $[0, \lambda]$.

Recall that for functions $f, g \in L^{1}(\mathbb{R})$, the convolution of $f$ and $g$ is defined (for almost every $x \in \mathbb{R}$ ) by

$$
(f * g)(x)=\int_{-\infty}^{\infty} f(x-y) g(y) \mathrm{d} y .
$$

Using convolution of functions, formula (8), which gives the PST, can be rewritten in the following form.

Proposition 3. For any function $f \in L^{1}(\mathbb{R})$ and any $0<s \leq \lambda$, we have

$$
\mathrm{PS}_{g} f(s, \lambda)=\sum_{n=-\infty}^{\infty}\left(f * \tilde{g}_{s}\right)(n \lambda)-\frac{s}{\lambda} \int_{-\infty}^{\infty} g(x) \mathrm{d} x \int_{-\infty}^{\infty} f(x) \mathrm{d} x .
$$

In the case where $\int_{-\infty}^{\infty} g(x) \mathrm{d} x=0$, we obtain

$$
\mathrm{PS}_{g} f(s, \lambda)=\sum_{n=-\infty}^{\infty}\left(f * \tilde{g}_{s}\right)(n \lambda) .
$$

3.5. The Calculus of the PST. In this section, we calculate the PST of the derivative $\partial^{k} f$ of a function $f$, for any positive integer $k$.

Theorem 4. Let $g$ be a fundamental function such that $\int_{-\infty}^{\infty} g(x) d x=0$, and let $f \in L^{1}(\mathbb{R})$ and $k$ be a positive integer. We assume that $f, g$ are differentiable up to $k$ times and the derivatives satisfy the following:

(i) $\partial^{i} f$ belongs to $L^{1}(\mathbb{R})$, for any $1 \leq i \leq k$.

(ii) $\partial^{i} g$ satisfies a decay condition similar to (1), for any $1 \leq i \leq k$.

Then, the PST of $\partial^{k} f$ is given by

$$
\operatorname{PS}_{g}\left(\partial^{k} f\right)=\left(\frac{-1}{s}\right)^{k} \operatorname{PS}_{\partial^{k} g}(f) .
$$


Proof. By the definition of the PST, we obtain

$$
\operatorname{PS}_{g}\left(\partial^{k} f\right)(s, \lambda)=\int_{-\infty}^{\infty} \partial^{k} f(x)\left(\sum_{n=-\infty}^{\infty} g\left(\frac{x-n \lambda}{s}\right)\right) \mathrm{d} x=\sum_{n=-\infty}^{\infty} \int_{-\infty}^{\infty} \partial^{k} f(x) g\left(\frac{x-n \lambda}{s}\right) \mathrm{d} x
$$

Integrating by parts $k$ times gives that

$$
\begin{aligned}
\operatorname{PS}_{g}\left(\partial^{k} f\right)(s, \lambda) & =\sum_{n=-\infty}^{\infty}(-1)^{k} \int_{-\infty}^{\infty} f(x) \partial^{k}\left[g\left(\frac{x-n \lambda}{s}\right)\right] \mathrm{d} x \\
& =\sum_{n=-\infty}^{\infty}(-1)^{k} \int_{-\infty}^{\infty} f(x) \frac{1}{s^{k}}\left(\partial^{k} g\right)\left(\frac{x-n \lambda}{s}\right) \mathrm{d} x \\
& =\left(\frac{-1}{s}\right)^{k} \int_{-\infty}^{\infty} f(x) \sum_{n=-\infty}^{\infty}\left(\partial^{k} g\right)\left(\frac{x-n \lambda}{s}\right) \mathrm{d} x \\
& =\left(\frac{-1}{s}\right)^{k} \operatorname{PS}_{\partial^{k} g}(f)(s, \lambda) .
\end{aligned}
$$

Remark 2. If we are not interested in much generality, we may assume that $f, g$ belong to the Schwartz class of functions. Recall that a function $f: \mathbb{R} \longrightarrow \mathbb{C}$ is called a Schwartz function if $f$ is infinitely differentiable and for any pair of nonnegative integers $k$ and $n$, we have

$$
\sup _{x \in \mathbb{R}}\left|x^{n} \partial^{k} f(x)\right|<\infty \text {. }
$$

Clearly, if $f, g$ are Schwartz functions, then the conditions of the above theorem are fulfilled.

\section{The PST and Discretization: The Poisson Summation Formula}

It is well known that periodization and discretization are dual procedures in the following sense: the periodization of a function is completely determined by discrete values of the original function's Fourier transform. And, conversely, the periodization of a function's Fourier transform is determined by discrete values of the original function. This statement becomes accurate with the Poisson summation formula (see [15] Theorem 1.17 or [16] Corollary 2.6 and also [17-19]).

Theorem 5 (Poisson summation formula). Assume that $f, \widehat{f} \in L^{1}(\mathbb{R})$ satisfy

$$
|f(x)| \leq \frac{C}{(1+|x|)^{1+\varepsilon}} \text { and }|\widehat{f}(x)| \leq \frac{C}{(1+|x|)^{1+\varepsilon}}
$$

for some $C, \varepsilon>0$. Then, both $f$ and $\hat{f}$ are continuous and for all $x \in \mathbb{R}$, we have

$$
\sum_{n=-\infty}^{\infty} \widehat{f}(n) e^{2 \pi i n x}=\sum_{n=-\infty}^{\infty} f(x+n)
$$

and in particular $\sum_{n=-\infty}^{\infty} \hat{f}(n)=\sum_{n=-\infty}^{\infty} f(n)$.

Applying Poisson's formula to the function $h(x)=f$ $(a x), a \neq 0$, and using the simple fact that $\widehat{h}(\omega)=1 / a \widehat{f}(\omega / a)$, we immediately get the following corollary.

Corollary 1. If $f$ and $\widehat{f}$ satisfy the conditions of the previous theorem, then for any $a \neq 0$ and any $x \in \mathbb{R}$, we have that

$$
\sum_{n=-\infty}^{\infty} f(a n)=\frac{1}{a} \sum_{n=-\infty}^{\infty} \hat{f}\left(\frac{n}{a}\right)
$$

Since the construction of PST is based on the periodization of a fundamental function after changing its scale, it is not surprising that $\operatorname{PS}_{g} f$ is determined by discrete samples of the Fourier transforms of the original function $f$ and the function $g$. More precisely, we prove the following result.

Theorem 6. Assume that the fundamental function $g$ satisfies the conditions:

$$
|g(x)| \leq \frac{C}{(1+|x|)^{2(1+\varepsilon)}} \text { and }|\widehat{g}(x)| \leq \frac{C}{(1+|x|)^{1+\varepsilon}}
$$

where $C, \varepsilon$ are positive constants. Let also $f \in L^{1}(\mathbb{R})$ be a function such that

$$
|f(x)| \leq \frac{C}{(1+|x|)^{1+\varepsilon}}
$$

Then, the PST of $f$ with respect to $g$ can be written in the form:

$\mathrm{PS}_{g} f(s, \lambda)=\frac{s}{\lambda} \sum_{n=-\infty}^{\infty} \hat{f}\left(\frac{n}{\lambda}\right) \hat{g}\left(\frac{-s n}{\lambda}\right)-\frac{s}{\lambda} \int_{-\infty}^{\infty} g(x) \mathrm{d} x \int_{-\infty}^{\infty} f(x) \mathrm{d} x$.

In particular, if $\int_{-\infty}^{\infty} g(x) \mathrm{d} x=0$, then we obtain

$$
\operatorname{PS}_{g} f(s, \lambda)=\frac{s}{\lambda} \sum_{n=-\infty}^{\infty} \widehat{f}\left(\frac{n}{\lambda}\right) \hat{g}\left(\frac{-s n}{\lambda}\right) \text {. }
$$

Proof. Firstly, we observe that 


$$
\begin{aligned}
|(f * g)(x)| & \leq \int_{-\infty}^{\infty}|f(x-y)||g(y)| \mathrm{d} y \leq \int_{-\infty}^{\infty} \frac{C}{(1+|x-y|)^{1+\varepsilon}}|g(y)| \mathrm{d} y \leq \int_{-\infty}^{\infty} \frac{C(1+|y|)^{1+\varepsilon}}{(1+|x|)^{1+\varepsilon}}|g(y)| \mathrm{d} y \\
& \leq \frac{C}{(1+|x|)^{1+\varepsilon}} \int_{-\infty}^{\infty} \frac{(1+|y|)^{1+\varepsilon}}{(1+|y|)^{2(1+\varepsilon)}} \mathrm{d} t \leq \frac{C}{(1+|x|)^{1+\varepsilon}}
\end{aligned}
$$

where $C$ denotes a constant (not always the same). Furthermore,

$$
|\mathscr{F}(f * g)(x)|=|\mathscr{F}(f)(x)| \cdot|\mathscr{F}(g)(x)| \leq f_{L^{1}} \frac{C}{(1+|x|)^{1+\varepsilon}} .
$$

Therefore, the assumptions on the functions $f$ and $g$ show that the convolution product $f * g$ satisfies the condition of Theorem 5 and Poisson's formula can be applied. Since for any (fixed) $s>0$ the function $\tilde{g}_{s}$ satisfies similar assumptions to the function $g$, we can also apply Poisson's formula to the product $f * \widetilde{g}_{s}$.
Now we recall the form of the PST which exploits the convolution of functions and is given in Proposition 3. Hence, we have

$$
\operatorname{PS}_{g} f(s, \lambda)=\sum_{n=-\infty}^{\infty}\left(f * \widetilde{g}_{s}\right)(n \lambda)-\frac{s}{\lambda} \int_{-\infty}^{\infty} g(x) \mathrm{d} x \int_{-\infty}^{\infty} f(x) \mathrm{d} x .
$$
that

Applying Poisson's formula to the function $f * \widetilde{g}_{s}$ gives

$$
\begin{aligned}
\sum_{n=-\infty}^{\infty}\left(f * \widetilde{g}_{s}\right)(n \lambda) & =\frac{1}{\lambda} \sum_{n=-\infty}^{\infty} \mathscr{F}\left(f * \widetilde{g}_{s}\right)\left(\frac{n}{\lambda}\right)=\frac{1}{\lambda} \sum_{n=-\infty}^{\infty} \mathscr{F}(f)\left(\frac{n}{\lambda}\right) \mathscr{F}\left(\widetilde{g}_{s}\right)\left(\frac{n}{\lambda}\right) \\
& =\frac{s}{\lambda} \sum_{n=-\infty}^{\infty} \mathscr{F}(f)\left(\frac{n}{\lambda}\right) \mathscr{F}(\tilde{g})\left(\frac{s n}{\lambda}\right)=\frac{s}{\lambda} \sum_{n=-\infty}^{\infty} \mathscr{F}(f)\left(\frac{n}{\lambda}\right) \mathscr{F}(g)\left(\frac{-s n}{\lambda}\right),
\end{aligned}
$$

and the result follows.

\section{Limit Behaviour}

This section is entirely devoted to the study of the behaviour of the PST for the limit values of the scale $s$ and the period $\lambda$.

Theorem 7. For any function $f \in L^{1}(\mathbb{R})$ and any $s>0$, the following holds:

$$
\lim _{\lambda \longrightarrow \infty} \operatorname{PS}_{g} f(s, \lambda)=\int_{-\infty}^{\infty} f(x) g\left(\frac{x}{s}\right) \mathrm{d} x .
$$

Lemma 2. For any $x \in \mathbb{R}$ and any $s>0$, we have

$$
\lim _{\lambda \longrightarrow \infty} G(x ; s, \lambda)=g\left(\frac{x}{s}\right) .
$$

Proof. Without loss of generality, we may assume that $\lambda>2|x|$. Then, using the decay condition (1), we can easily obtain

$$
\left|G(x ; s, \lambda)-g\left(\frac{x}{s}\right)\right| \leq \sum_{n \in \mathbb{Z}, n \neq 0}\left|g\left(\frac{x-n \lambda}{s}\right)\right| \leq 2 C \frac{s^{1+\varepsilon}}{\lambda^{1+\varepsilon}} \sum_{n=1}^{\infty} \frac{1}{(n-1 / 2)^{1+\varepsilon}}
$$

and the result follows.

Proof of Theorem 7. By inequality (4), for $0<s \leq \lambda$, the family of functions $G(\cdot ; s, \lambda)$ is bounded by some absolute constant $C$. Therefore, $f(x) G(x ; s, \lambda)$ is dominated by the integrable function $C f(x)$. The dominated convergence theorem and the previous lemma show now that

$$
\lim _{\lambda \longrightarrow \infty} \int_{-\infty}^{\infty} f(x) G(x ; s, \lambda) \mathrm{d} x=\int_{-\infty}^{\infty} \lim _{\lambda \longrightarrow \infty} f(x) G(x ; s, \lambda) \mathrm{d} x=\int_{-\infty}^{\infty} f(x) g\left(\frac{x}{s}\right) \mathrm{d} x .
$$

Clearly,

$$
\lim _{\lambda \longrightarrow \infty} \frac{s}{\lambda} \int_{-\infty}^{\infty} g(x) \mathrm{d} x \int_{-\infty}^{\infty} f(x) \mathrm{d} x=0,
$$

and the proof of the theorem is complete.
Theorem 8. For any function $f \in L^{1}(\mathbb{R})$ and any $\lambda>0$, the following holds:

$$
\lim _{s \longrightarrow 0^{+}} \operatorname{PS}_{g} f(s, \lambda)=0 .
$$




$$
\lim _{s \longrightarrow 0^{+}} G(x ; s, \lambda)=G_{0}(x ; \lambda),
$$

where

$$
G_{0}(x ; \lambda)= \begin{cases}g(0), & \text { if } x=k \lambda, k \in Z \\ 0, & \text { elsewhere }\end{cases}
$$

Proof. Since $G$ is periodic with period $\lambda$, it suffices to prove the lemma for any $x \in[0, \lambda)$. We first consider the case $x=0$. Then, an easy calculation exploiting the decay condition (1) gives that

$$
|G(0 ; s, \lambda)-g(0)|=\left|\sum_{n \in \mathbb{Z}, n \backslash n e 0} g\left(\frac{n \lambda}{s}\right)\right| \leq 2 C \frac{s^{1+\varepsilon}}{\lambda^{1+\varepsilon}} \sum_{n=1}^{\infty} \frac{1}{n^{1+\varepsilon}} .
$$

Hence, it follows that $\lim _{s \longrightarrow 0^{+}} G(0 ; s, \lambda)=g(0)$.

We next consider the case where $x \in(0, \lambda)$. Since $s \longrightarrow 0^{+}$, we may assume, without loss of generality, that $x \in(\sqrt{s}, \lambda-\sqrt{s})$. Then, we have

$$
\begin{aligned}
|G(x ; s, \lambda)| \leq & \sum_{n=-\infty}^{\infty} \frac{C s^{1+\varepsilon}}{(s+|x-n \lambda|)^{1+\varepsilon}} \\
= & \frac{C s^{1+\varepsilon}}{(s+|x|)^{1+\varepsilon}}+\frac{C s^{1+\varepsilon}}{(s+|x-\lambda|)^{1+\varepsilon}} \\
& +\sum_{n \in \mathbb{Z}, n \neq 0,1} \frac{C s^{1+\varepsilon}}{(s+|x-n \lambda|)^{1+\varepsilon}} \\
\leq & 2 C(\sqrt{s})^{1+\varepsilon}+2 C \frac{s^{1+\varepsilon}}{\lambda^{1+\varepsilon}} \sum_{n=1}^{\infty} \frac{1}{n^{1+\varepsilon}} .
\end{aligned}
$$

Therefore, $\lim _{s \longrightarrow 0^{+}} G(x ; s, \lambda)=0$ and this completes the proof of the lemma.

Proof of Theorem 8. As in the Proof of Theorem 7, the dominated convergence theorem and the previous lemma imply that

$$
\lim _{s \longrightarrow 0^{+}} \int_{-\infty}^{\infty} f(x) G(x ; s, \lambda) \mathrm{d} x=\int_{-\infty}^{\infty}(x) G_{0}(x ; \lambda) \mathrm{d} x .
$$

Since $G_{0}$ is zero for almost every $x \in \mathbb{R}$, we have

$$
\lim _{s \longrightarrow 0^{+}} \int_{-\infty}^{\infty} f(x) G(x ; s, \lambda) \mathrm{d} x=\int_{-\infty}^{\infty} f(x) G_{0}(x ; \lambda) \mathrm{d} x=0 .
$$

Clearly,

$$
\lim _{s \longrightarrow 0^{+}} \frac{s}{\lambda} \infty \int_{-\infty}^{\infty} g(x) \mathrm{d} x \int_{-\infty}^{\infty} f(x) \mathrm{d} x=0,
$$

and the desired result follows.

It remains to investigate the behaviour of the PST when the period $\lambda$ tends to zero. This means that the inverse of period, that is, the frequency, tends to infinity. The well- known Riemann-Lebesgue lemma asserts that the FT, $\widehat{f}(\omega)$, of a function $f \in L^{1}(\mathbb{R})$ converges to zero, when the frequency $|\omega| \longrightarrow \infty$. Since the FT can be derived from the PST (see the next section), it is reasonable to expect that the PST of an $L^{1}$ function converges to 0 as $\lambda$ goes to 0 . This is true; however, we have to impose some further restrictions on the functions $f$ and $g$.

Theorem 9. Assume that the fundamental function $g$ satisfies the conditions:

$$
\int_{-\infty}^{\infty} g(x) \mathrm{d} x=0,|g(x)| \leq \frac{C}{(1+|x|)^{2(1+\varepsilon)}} \text { and }|\hat{g}(x)| \leq \frac{C}{(1+|x|)^{1+\varepsilon}} .
$$

Let also $f \in L^{1}(\mathbb{R})$ be a function such that

$$
|f(x)| \leq \frac{C}{(1+|x|)^{1+\varepsilon}} \text { and }\left|x^{2} \widehat{f}(x)\right| \leq C .
$$

For example, $f, g$ may be Schwartz functions. Then, we have that

$$
\lim _{\lambda \longrightarrow 0^{+}} \operatorname{PS}_{g} f(s, \lambda)=0 .
$$

Proof. By Theorem 6, the PST of $f$ is given by discrete samples of the FTs of $f$ and $g$, namely:

$$
\operatorname{PS}_{g} f(s, \lambda)=\frac{s}{\lambda} \sum_{n=-\infty}^{\infty} \widehat{f}\left(\frac{n}{\lambda}\right) \widehat{g}\left(\frac{-s n}{\lambda}\right)
$$

By our assumptions on the FT of $f$, it follows that for any $\lambda \in(0,1)$ and any $n \neq 0$ :

$$
\left|\widehat{f}\left(\frac{n}{\lambda}\right) \hat{g}\left(\frac{-s n}{\lambda}\right)\right| \leq \frac{C}{n^{2}}\|g\|_{L^{1}}
$$

Therefore, the series in (59) converges uniformly for $0<s \leq \lambda<1$ and thus

$$
\lim _{\lambda \longrightarrow 0^{+}} \operatorname{PS}_{g} f(s, \lambda)=\sum_{n=-\infty}^{\infty} \lim _{\lambda \longrightarrow 0} \frac{s}{\lambda} \widehat{f}\left(\frac{n}{\lambda}\right) \hat{g}\left(\frac{-s n}{\lambda}\right) .
$$

However, for $n=0$, we have

$$
\widehat{g}(0)=\int_{-\infty}^{\infty} g(x) \mathrm{d} x=0
$$

and for $n \neq 0$,

$$
\left|\frac{s}{\lambda} \widehat{f}\left(\frac{n}{\lambda}\right) \hat{g}\left(\frac{-s n}{\lambda}\right)\right| \leq\left|\widehat{f}\left(\frac{n}{\lambda}\right)\right|\|g\|_{L^{1}} \leq \frac{C \lambda^{2}}{n^{2}}\|g\|_{L^{1}} .
$$

It follows that 


$$
\lim _{\lambda \longrightarrow 0^{+}} \operatorname{PS}_{g} f(s, \lambda)=0
$$

\section{Connection with Other Transforms}

6.1. Connection with the FT. The connection between the PST and the FT has already become clear by the Poisson summation formula (Theorem 6). The purpose of this section is to show that the FT can be derived as a special case of the PST.

Consider the fundamental function $g(x)=\exp (-2 \pi$ $i x)=e^{-2 \pi i x}, 0 \leq x<1$. Then, $g(x / s)=\exp (-2 \pi i x / s)$, for $0 \leq$ $x<s$, and it is not hard to see that $G(x ; s, s)=\exp (-2 \pi i x / s)$ for any $x \in \mathbb{R}$. Furthermore,

$$
\int_{-\infty}^{\infty} g(x) \mathrm{d} x=\int_{0}^{1} e^{-2 \pi i x} \mathrm{~d} x=0
$$

Consequently, for any function $f \in L^{1}(\mathbb{R})$, we have

$$
\operatorname{PS}_{g} f(s, s)=\int_{\mathbb{R}} f(x) \exp \left(-\frac{2 \pi i x}{s}\right) \mathrm{d} x=\mathscr{F} f\left(\frac{1}{s}\right) \text {, }
$$

that is, the diagonal of PST gives the FT of $f$ for the positive frequencies.

In a similar way, we can produce the inverse Fourier transform. Indeed, we take the fundamental function $g(x)=\exp (2 \pi i x)=e^{2 \pi i x}, 0 \leq x<1$. Then, $G(x ; s, s)=\exp$ $(2 \pi i x / s)$ for any $x \in \mathbb{R}$ and,

$$
\operatorname{PS}_{g} f(s, s)=\int_{\mathbb{R}} f(x) \exp \left(\frac{2 \pi i x}{s}\right) \mathrm{d} x=\mathscr{F}^{-1} f\left(\frac{1}{s}\right) .
$$

6.2. Connection with the WT. A wavelet is a function $\psi \in L^{1}(\mathbb{R})$ such that $\int_{-\infty}^{\infty} \psi(x) \mathrm{d} x=0$. We point out that the term wavelet does not possess a unique definition. However, the above definition is appropriate and for all practical purposes. Given the wavelet $\psi$ and a function $f \in L^{\infty}(\mathbb{R})$, the non-normalized continuous wavelet transform (WT) of $f$ with respect to $\psi$ is defined as

$$
W_{\psi} f(s, t)=\int_{-\infty}^{\infty} f(x) \psi\left(\frac{x-t}{s}\right) \mathrm{d} x .
$$

Fundamental works on wavelet theory are $[7,8,20,21]$.

If the fundamental function $g$ of the PST is a wavelet, then equation (8) becomes

$$
\operatorname{PS}_{g} f(s, \lambda)=\sum_{n=-\infty}^{\infty} \int_{-\infty}^{\infty} f(x) g\left(\frac{x-n \lambda}{s}\right) \mathrm{d} x=\sum_{n=-\infty}^{\infty} W_{g} f(s, n \lambda) .
$$

That is, the PST is an infinite sum of wavelet transforms of $f$.

Furthermore, it follows, by Theorem 7, that

$$
\lim _{\lambda \longrightarrow \infty} \operatorname{PS}_{g} f(s, \lambda)=\int_{-\infty}^{\infty} f(x) g\left(\frac{x}{s}\right) \mathrm{d} x=W_{g} f(s, 0) .
$$

Therefore, if we replace the initial function $g$ with its translation $\tau_{t} g(x)=g(x-t)$, then the above observation implies that

$$
\lim _{\lambda \longrightarrow \infty} \operatorname{PS}_{\tau_{t} g} f(s, \lambda)=\int_{-\infty}^{\infty} f(x) \tau_{t} g\left(\frac{x}{s}\right)=W_{g} f(s, t s) .
$$

Remark 3. Summarizing the results of Sections 6.1 and 6.2, we can say that the PST unifies the two well-known and extensively studied transforms: the FT and the WT. Roughly speaking, these two transforms correspond to the two extreme cases of the PST. The case $s=\lambda$ corresponds to the FT, while the case $\lambda=\infty$ gives the WT.

6.3. Connection with the Laplace Transform. In view of Theorem 7, we observe that the limit values of PST as $\lambda$ tends to infinity can produce many other integral transforms, that is, transforms of the form $\int f(x) K(x, s) \mathrm{d} x$ which are defined by a kernel $K(x, s)$. Take for example the (one-sided) Laplace transform defined by

$$
(\mathscr{L} f)(s)=\int_{0}^{\infty} f(x) \exp (-s x) \mathrm{d} x .
$$

Using the fundamental function $g(x)=e^{-x}$, if $x>0$ and $g(x)=0$ elsewhere, it is not hard to conclude that for any $s>0$,

$$
\lim _{\lambda \longrightarrow \infty} \operatorname{PS}_{g} f(s, \lambda)=(\mathscr{L} f)\left(\frac{1}{s}\right)
$$

\section{The PST of Some Selected Functions}

The purpose of the present section is to illustrate the PST to some selected functions and real 1D surfaces.

7.1. The PST of the Characteristic Function of an Interval. We start with the characteristic function of the interval $[0, T], T>0$, which is defined by

$$
f(x)=\chi_{[0, T]}(x)= \begin{cases}1, & 0 \leq x \leq T \\ 0, & \text { otherwise. }\end{cases}
$$

Then, for $0<s \leq \lambda$, formula (8) gives that

$$
\operatorname{PS}_{g} f(s, \lambda)=\sum_{n=-\infty}^{\infty} \int_{0}^{T} g\left(\frac{x-n \lambda}{s}\right) \mathrm{d} x-\frac{s T}{\lambda} \int_{-\infty}^{\infty} g(x) \mathrm{d} x .
$$

In order to complete the above calculation, we will use several fundamental functions. Firstly, we consider the function $g(x)=\chi_{[0,1]}(x)$. Then, for $0<s<\lambda$, it follows that

$$
\mathrm{PS}_{g} f(s, \lambda)=f(x)=\left\{\begin{array}{l}
\left(\left[\frac{T}{\lambda}\right]+1\right) s-\frac{s}{\lambda} T, \quad \frac{s}{\lambda} \leq \frac{T}{\lambda}-\left[\frac{T}{\lambda}\right]<1, \\
{\left[\frac{T}{\lambda}\right](s-\lambda)+T-\frac{s}{\lambda} T, 0 \leq \frac{T}{\lambda}-\left[\frac{T}{\lambda}\right]<\frac{s}{\lambda},}
\end{array}\right.
$$

where for any real number $x,[x]$ denotes the integer part of $x$. 
Secondly, we consider the fundamental function $g(x)=e^{-2 \pi i x}$, for $0 \leq x<1$. Then, $\int_{-\infty}^{\infty} g(x) \mathrm{d} x=0$ and for $s<\lambda$, it follows that

$$
\mathrm{PS}_{g} f(s, \lambda)= \begin{cases}\frac{s}{\pi} \exp \left(-\pi i \frac{T-[T / \lambda]}{s}\right) \sin \left(\pi \frac{T-[T / \lambda]}{s}\right), & 0 \leq \frac{T}{\lambda}-\left[\frac{T}{\lambda}\right]<\frac{s}{\lambda}, \\ 0, & \frac{s}{\lambda} \leq \frac{T}{\lambda}-\left[\frac{T}{\lambda}\right]<1 .\end{cases}
$$

As it was expected, the above formula is close to the FT of $f$ which is given by

$$
\widehat{f}(\omega)=\frac{1}{\pi \omega} \exp (-\pi i T \omega) \sin (\pi T \omega) .
$$

Finally, we take the fundamental function $g(x)=\sin x$, for $0 \leq x<1$. Then, for $s<\lambda$, we have

$$
\mathrm{PS}_{g} f(s, \lambda)= \begin{cases}\frac{s}{2 \pi}\left(1-\cos \left(2 \pi \frac{T-[T / \lambda]}{s}\right)\right), & 0 \leq \frac{T}{\lambda}-\left[\frac{T}{\lambda}\right]<\frac{s}{\lambda}, \\ 0, & \frac{s}{\lambda} \leq \frac{T}{\lambda}-\left[\frac{T}{\lambda}\right]<1 .\end{cases}
$$

7.2. The PSTof the Gaussian. We continue with the Gaussian function

$$
f(x)=\frac{1}{\sigma \sqrt{2 \pi}} \exp \left(-\frac{(x-\mu)^{2}}{2 \sigma^{2}}\right)
$$

with mean value $\mu$ and standard deviation $\sigma^{2}$. We also take as a fundamental function the Gaussian

$$
g(x)=\frac{1}{\sqrt{2 \pi}} \exp \left(-\frac{x^{2}}{2}\right)
$$

with $\mu=0$ and $\sigma^{2}=1$. Then, the PS transform of the function $f$ is given by

$$
\operatorname{PS}_{g} f(s, \lambda)=\frac{1}{\sqrt{2 \pi}} \cdot \frac{s}{\sqrt{s^{2}+\sigma^{2}}} \cdot \sum_{n \in \mathbb{Z}} \exp \left(-\frac{(n \lambda-\mu)^{2}}{2\left(s^{2}+\sigma^{2}\right)}\right)-\frac{s}{\lambda}
$$

7.3. The PST of a Rectangular Pulse Train. Consider the rectangular pulse train function defined by its period $P=50$ and width (duty cycle) $S=20$ shown in Figure 2(a) and described by the following formula:

$$
f(x)=\left\{\begin{array}{ll}
1, & n P<x<n P+s \\
0, & \text { elsewhere }
\end{array}, \quad n=0,1,2, \ldots\right.
$$

The PST of this function is calculated by using the kernel $G(x ; s, \lambda)$ of a rectangular pulse train with varied period $\lambda$ and feature width $s$ (see equation (7)):

$$
G(x ; \lambda, s)=\left\{\begin{array}{ll}
1, & n \lambda<x<n \lambda+s \\
0, & \text { elsewhere }
\end{array}, \quad n=0,1,2, \ldots\right.
$$

The resulting two-dimensional PST $\mathrm{PS}_{g} f(s, \lambda)$ is shown in Figure 2(b) for $1<s<50$ and $5<\lambda<120$. One can directly notice the presence of a strong peak at $\lambda=P=50$, while less pronounced peaks also appear at the half and double periods, i.e., at $\lambda=P / 2=25$ and $\lambda=2 P=100$. Similar peaks are expected at higher multiples or lower submultiples of the fundamental period $(\lambda=150,200, \ldots)$ if these periods were included in the diagram of Figure 2(b). The ordinate value of all peaks is at $s$ very close to 20 as also shown in the inset diagram where the $1 \mathrm{D}$ section of PST at $\lambda=50\left(\mathrm{PS}_{g} f(s, \lambda)=\right.$ $50)$ is illustrated. Therefore, this simple example of periodic function reveals that PST is able to quantify both period and feature scale simultaneously.

7.4. The PST of a Gaussian Pulse Train. A little more complicated example of $1 \mathrm{D}$ surface is the Gaussian pulse train function comprising Gaussian peaks with fixed width arranged at periodic positions as shown below:

$$
f(x)=\sum_{n} \exp \left(-\frac{(x-n P)^{2}}{2 S^{2}}\right) .
$$

An illustration of such a 1D surface is shown in Figure 3(a) where the width of the repeated Gaussian is $S=$ 10 while the period $P=80$.

The PST is calculated using definition (7) with the kernel function $G$ consisting of periodically arranged Gaussian peaks with varying period $\lambda$ and scale $s$ :

$$
G(x ; s, \lambda)=\sum_{m} \exp \left(-\frac{(x-m \lambda)^{2}}{2 s^{2}}\right) .
$$

Figure 3(b) shows $\mathrm{PS}_{g} f$ which is dominated by the presence of two peaks at $s=10$ and $\lambda=80$ and $\lambda=160$, respectively, while PST is almost zero elsewhere. The first peak at $(s=10, \lambda=80)$ corresponds to the period and scale of the input pulse train and the second is the first harmonic at $\lambda=2 \cdot 80=160$. The inset of Figure $3(\mathrm{~b})$ magnifies the $1 \mathrm{D}$ behaviour of PST for $\lambda=80$ versus scale $s$.

The above simple deterministic examples show that (a) PST is a sufficient tool to reveal simultaneously both the period and scale of 1D surfaces and (b) PST is characterized by distinct 


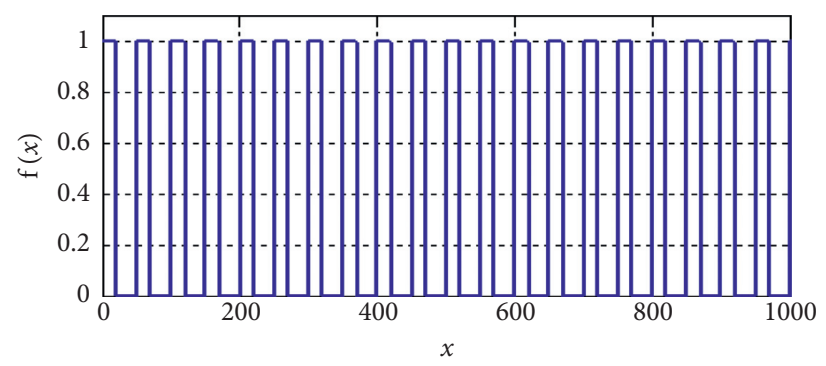

(a)

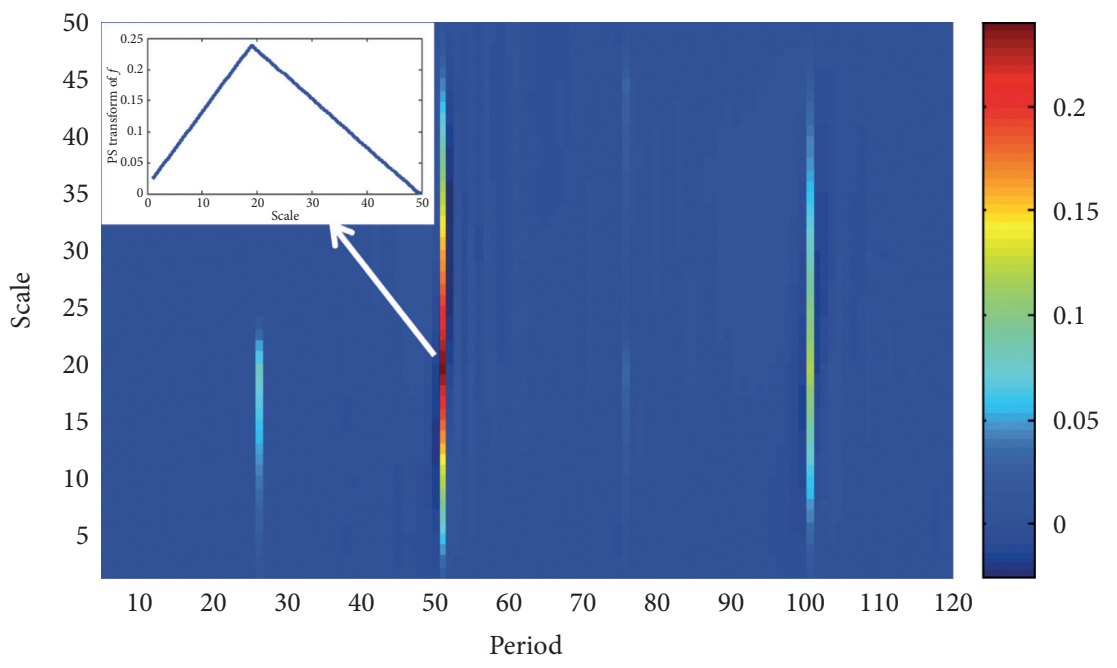

(b)

Figure 2: A rectangular pulse wave with period $=50$ and duty cycle (pulse scale) $=20$ (a) along with its PST (b). The inset magnifies the transform function at $\lambda=50$ versus scale/width. One can notice the direct way the peaks in PST reveal simultaneously both period and duty cycle (scale) of the analyzed function.

peaks with asymmetrical structure since they are much steeper along period axis (horizontally) than scale axis (vertically).

One can readily observe the asymmetry in the steepness of the peaks: along period axis they are much steeper than scale axis.

7.5. The PST of an Experimental 1D Surface. In order to get a more realistic example of PST application, we consider a polymer surface treated with oxygen plasma for 1 minute inside a low temperature plasma reactor. The surface topography is measured by an atomic force microscope (AFM) in a grid of $512 \times 512$ points while the total measured area is $2 \times 2 \mu \mathrm{m}^{2}$. The experimental background and measurement details can be found elsewhere [22]. A randomly selected profile $f(x)$ of this surface is displayed in Figure 4(a). Its morphology is roughly characterized by the presence of more or less well-defined peaks which are distributed along profile with an apparently random manner. However, the PSD analysis shown in Figure 4(b) reveals the existence of several periodicities manifested by the presence of peaks at specific frequencies. The smooth peak A with the highest frequency at $0.02 \mathrm{~nm}^{-1}$ corresponds to the smaller period of $50 \mathrm{~nm}$ which roughly quantifies the mean distance between nearby peaks when all peaks (high and low) are considered. However, the peaks are diversified with respect to their scales (height and width) and the higher ones define new approximate periodicities with larger periods on the background of the fundamental period of $50 \mathrm{~nm}$. These periods are manifested by the peaks B-E in PSD and reflect periodicities of approximately $80,140,155$, and $225 \mathrm{~nm}$, respectively. Therefore, the profile morphology is characterized by multiperiodicity induced by the multiscaling of peak dimensions and especially their heights. However, the multiscale nature of peaks cannot be fully quantified by the PSD analysis since the information about their widths is missing. On the other hand, Figure 4(c) shows the PST of the same profile using as kernel a Gaussian pulse train similar to those used in the Section 7.4. As expected, PST reproduces the periodicities detected by PSD at 50, 75, 140,160 , and $225 \mathrm{~nm}$, but at the same time, it proceeds one step further to characterize the scales (i.e., widths) of the peaks involved in each periodicity. Thus, for example, the fundamental period of $50 \mathrm{~nm}$ is created by peaks of average width $8-10 \mathrm{~nm}$, while the largest periodic structure with period $225 \mathrm{~nm}$ includes bolder protrusions of $35 \mathrm{~nm}$ width each.

This example certifies the usefulness of PST analysis to disclose at the same diagram both hidden periodicities and feature scales in apparently random morphologies of profiles and surfaces. 


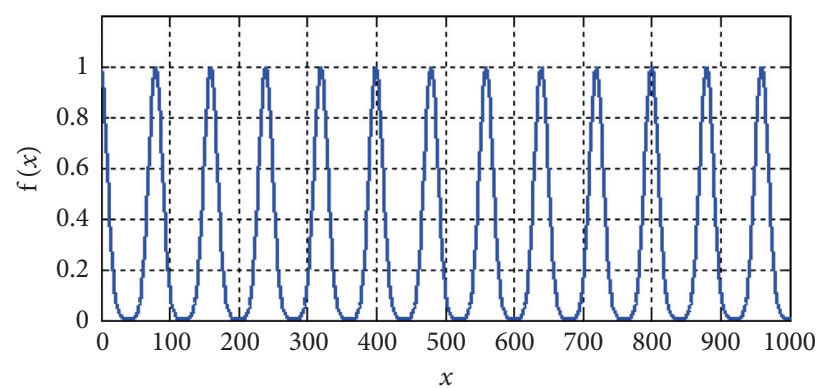

(a)
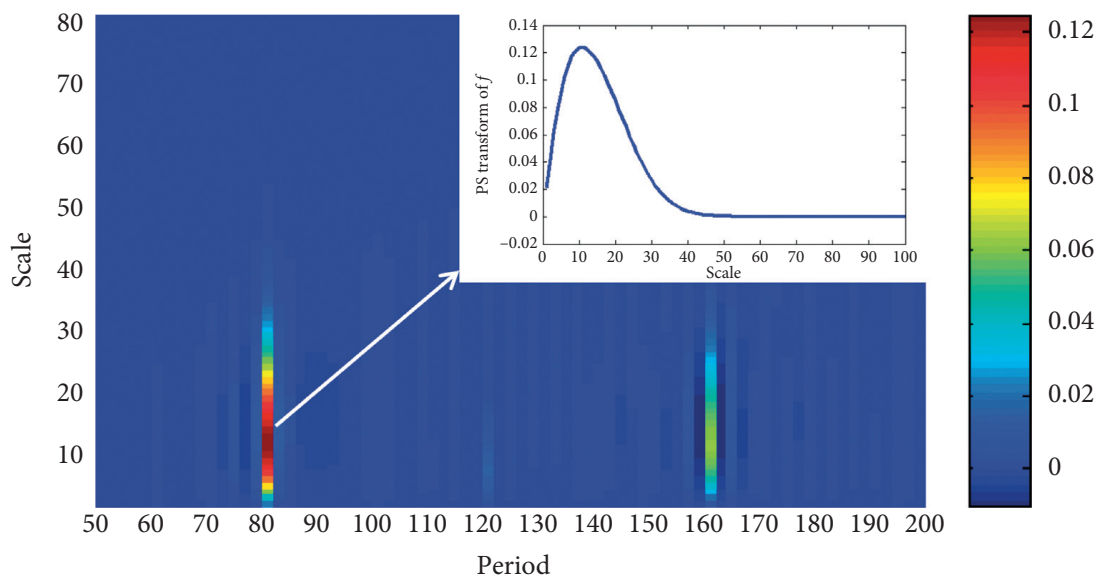

(b)

Figure 3: A Gaussian pulse wave with period $=80$ and width (scale) of repeated Gaussian equal to 20 (a) along with its PST (b). The inset magnifies the transform function at the period $\lambda=80$. Similar to Figure 2, the PST captures simultaneously both period and scale of the analyzed function.
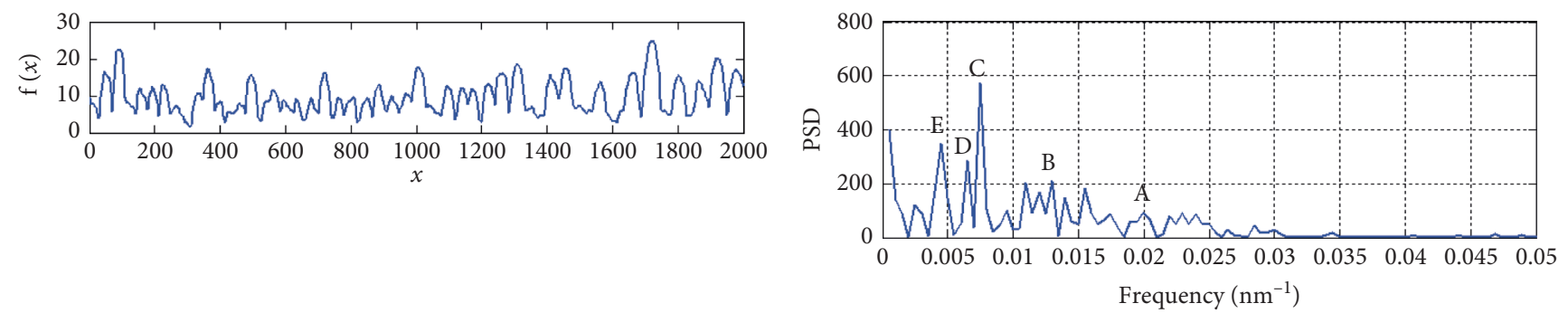

(a)

(b)

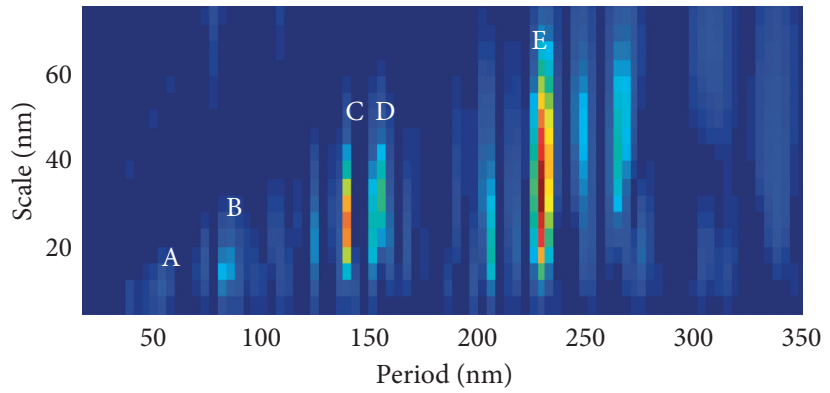

(c)

Figure 4: (a) A profile of a polymer surface etched in oxygen plasma treatment for $1 \mathrm{~min}$. Notice the multiperiodic and multiscale profile morphology. (b) The PSD of the profile exhibiting five regions (A-E) with more or less well-distinguished peaks (or group of peaks) characterizing hidden periodicities in the profile and (c) the PST of the profile using as kernel a Gaussian pulse train. It reproduces the PSD information about existing periodicities (see periods of A-E peaks) while additionally it outputs the lateral scales corresponding to the features of the detected periodicities (see widths of A-E). 


\section{Summary and Conclusions}

Fourier and wavelet transforms play a prominent role in several applications, e.g., signal analysis, surface measurement, etc. FT is the standard tool for analyzing periodic surfaces as it provides us with accurate information about the harmonic components of the analyzed surface. On the other hand, WT can detect irregularities of the surface such as cracks or scratches (see [23] for a discussion of the advantages and disadvantages of the use of FT and WT in the analysis of a geometrical surface structure).

It is noteworthy that several surfaces encountered in nature and technology are almost periodic and they can be viewed as generated by a feature repeated endlessly. Properties of such surfaces depend not only on period or irregularities but also on the width (or scale) of the repeated feature and, in many cases, on the ratio of period and scale. Despite the success of FT and WT in surface characterization, they are not able to quantify straightforwardly the feature scale and ratio.

Taking into account the above considerations, we have proposed and presented the period-scale transform. Its purpose is to detect simultaneously the harmonic components of the analyzed surface as well as the scale of the repeated feature.

The PST is an integral transform, i.e., it is of the form

$$
\mathrm{PS}_{g} f(s, \lambda)=\int_{-\infty}^{\infty} f(x)(G(x ; s, \lambda)-\backslash \operatorname{sca}\{G\}) \mathrm{d} x,
$$

where the kernel $G(\cdot ; s, \lambda)$ comes from the periodization with period $\lambda$ of a fundamental function $g$, with spatial scale $s$.

The PST defines a bounded linear operator. As far as scaling properties, the transform behaves very well with respect to dilation (Theorem 1) but it is not phase invariant (Theorem 2). Several other properties of the PST have been detailed in this work. Furthermore, we have addressed the limit behaviour of the PST and we have shown that PST bridges the gap between FT and WT in the sense that these two classical transforms can be realized as special cases of PST.

Finally, we have examined the application of PST to model and real 1D surfaces. It was found that PST provides valuable information for both period and feature scale aspects of surface morphology. For example, in the case of the experimental profile, we compared the outputs of PST and FT and explicitly showed the additional information about the scales of repeated surface features provided by PST in excess of FT.

Future work will explore more applications of PST to real surfaces and focus on the relationship with specific surface functionalities (light reflectance, wetting, friction, and so on). Also, more attention will be paid on the phase dependence of PST and its impact on the final result.

\section{Data Availability}

The data used to support the findings of this study are available from the corresponding author upon request.

\section{Conflicts of Interest}

The authors declare that they have no conflicts of interest.

\section{References}

[1] T. R. Thomas, Rough Surfaces, Imperial College Press, London, UK, 2nd edition, 1999.

[2] E. Gogolides, V. Constantoudis, G. Kokkoris et al., "Controlling roughness: from etching to nanotexturing and plasma-directed organization on organic and inorganic materials," Journal of Physics D: Applied Physics, vol. 44, no. 17, Article ID 174021, 2011.

[3] W.-K. Kuo, J.-J. Hsu, C.-K. Nien, and H. H. Yu, "Moth-eyeinspired biophotonic surfaces with antireflective and hydrophobic characteristics," ACS Applied Materials \& Interfaces, vol. 8, no. 46, pp. 32021-32030, 2016.

[4] T. Zhang, S. Han, and J. Zhou, "Dynamic behaviours for semidiscrete stochastic Cohen-Grossberg neural networks with time delays," Journal of the Franklin Institute, vol. 357, no. 17, pp. 13006-13040, 2020.

[5] T. Zhang and L. Xiong, "Periodic motion for impulsive fractional functional differential equations with piecewise Caputo derivative," Applied Mathematics Letters, vol. 101, Article ID 106072, 2020.

[6] T. Zhang, J. Zhou, and Y. Liao, "Exponentially stable periodic oscillation and mittag-leffler stabilization for fractional-order impulsive control neural networks with piecewise caputo derivatives," IEEE Transactions on Cybernetics, pp. 1-14. in press, 2021.

[7] G. Kaiser, A Friendly Guide to Wavelets, Birkhäuser Boston, Inc., Boston, MA, USA, 1994.

[8] I. Daubechies, Ten Lectures on Wavelets, Society for Industrial and Applied Mathematics (SIAM), Philadelphia PA, 1992.

[9] N. Y. Abed and O. A. Mohammed, "Modeling and characterization of transformers internal faults using finite element and discrete wavelet transforms," IEEE Transactions on Magnetics, vol. 43, no. 4, pp. 1425-1428, 2007.

[10] M. Kordestani, M. F. Samadi, and M. Saif, "A new hybrid fault prognosis method for MFS systems based on distributed neural networks and recursive bayesian algorithm," IEEE Systems Journal, vol. 14, no. 4, pp. 5407-5416, 2020.

[11] T. Li, Z. Zhao, C. Sun et al., "WaveletKernelNet: an interpretable deep neural network for industrial intelligent diagnosis," IEEE Transactions on Systems, Man, and Cybernetics: Systems, vol. 99, pp. 1-11, 2021.

[12] H. Shao, M. Xia, J. Wan, and C. De Silva, "Modified stacked auto-encoder using adaptive morlet wavelet for intelligent fault diagnosis of rotating machinery," IEEE/ASME Transactions on Mechatronics, p. 1. in press, 2021.

[13] M. Kordestani, M. Saif, M. E. Orchard, R. Razavi-Far, and K. Khorasani, "Failure prognosis and applications-A survey of recent literature," IEEE Transactions on Reliability, vol. 70, no. 2, pp. 728-748, 2021.

[14] J. Fischer, "On the duality of discrete and periodic functions," Mathematics, vol. 3, no. 2, pp. 299-318, 2015.

[15] L. Grafakos, "Classical Fourier analysis," Graduate Texts in Mathematics, Vol. 249, Springer, New York, 3rd edition, 2014.

[16] E. M. Stein and G. Weiss, Introduction to Fourier Analysis on Euclidean Spaces, Princeton University Press, Princeton, NJ, USA, 1971

[17] J. J. Benedetto, "Harmonic analysis and applications," Studies in Advanced Mathematics, CRC Press, Boca Raton, FL, USA, 1997. 
[18] J. J. Benedetto and G. Zimmermann, "Sampling multipliers and the Poisson summation formula," The Journal of Fourier Analysis and Applications, vol. 3, no. 5, pp. 505-523, 1997.

[19] C. Gasquet and P. Witomski, "Fourier analysis and applications. Filtering, numerical computation, wavelets," Texts in Applied Mathematics, Vol. 30, Springer-Verlag, New York, NY, USA, 1999.

[20] S. Mallat, A Wavelet Tour of Signal Processing, Academic Press, San Diego, CF, USA, 1998.

[21] Y. Meyer, Ondelettes et Opérateurs, Hermann, Paris, France, 1990.

[22] D. Kontziampasis, V. Constantoudis, and E. Gogolides, "Plasma directed organization of nanodots on polymers: effects of polymer type and etching time on morphology and order," Plasma Processes and Polymers, vol. 9, no. 9, pp. 866-872, 2012.

[23] S. Adamczak, W. Makiela, and K. Stepien, "Investigating advantages and disadvantages of the analysis of a geometrical surface structure with the use of Fourier and Wavelet transform," Metrology and Measurement Systems, vol. 17, no. 2, pp. 233-244, 2010. 